Slavica

bruxellensia

\section{Slavica bruxellensia}

Revue polyphonique de littérature, culture et histoire

slaves

9 | 2013

Érotisme

\title{
Entretien polyphonique sur l'érotisme et la sexualité dans les littératures slaves
}

Katia Vandenborre, Dorota Walczak, Blažina Dalibor, Ulrike Jekutsch, Maria Kistereva, Wiera Meniok, Joanna Partyka et Jan Rubeš

\section{OpenEdition}

\section{Journals}

\section{Édition électronique}

URL : https://journals.openedition.org/slavica/1395

DOI : 10.4000/slavica.1395

ISSN : 2034-6395

\section{Éditeur}

Université libre de Bruxelles - ULB

\section{Référence électronique}

Katia Vandenborre, Dorota Walczak, Blažina Dalibor, Ulrike Jekutsch, Maria Kistereva, Wiera Meniok, Joanna Partyka et Jan Rubeš, « Entretien polyphonique sur l'érotisme et la sexualité dans les littératures slaves », Slavica bruxellensia [En ligne], 9 | 2013, mis en ligne le 15 avril 2013, consulté le 16 septembre 2022. URL : http://journals.openedition.org/slavica/1395 ; DOI : https://doi.org/10.4000/ slavica. 1395

Ce document a été généré automatiquement le 16 septembre 2022.

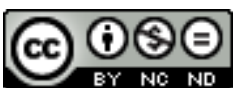

Creative Commons - Attribution - Pas d'Utilisation Commerciale - Pas de Modification 4.0 International - CC BY-NC-ND 4.0

https://creativecommons.org/licenses/by-nc-nd/4.0/ 


\title{
Entretien polyphonique sur l'érotisme et la sexualité dans les littératures slaves
}

\author{
Katia Vandenborre, Dorota Walczak, Blažina Dalibor, Ulrike Jekutsch, Maria
} Kistereva, Wiera Meniok, Joanna Partyka et Jan Rubeš

\section{Présentation}

Comment l'érotisme se définit-il aujourd'hui? Quelle est la place de l'érotisme dans la recherche scientifique en littérature slave ? Y-a-t-il des moments plus propices que d'autres pour explorer ce sujet dans l'histoire des littératures et des sociétés? Le tabou constitue-t-il une notion fondamentale dans l'étude de l'érotisme et de la sexualité ? Comment les changements de mœurs et d'expression littéraire des auteurs slaves contemporains se déclinent-ils face aux attentes des lecteurs en termes d'originalité et de nouveauté ? Ces questions et bien d'autres, nous les avons posées d'une manière libre et non exhaustive à quelques chercheurs et professeurs de divers pays et universités, qui ont eu le courage et l'énergie d'y répondre et de mener ainsi une réflexion sur le sujet. Afin de rendre compte des tendances littéraires et scientifiques qui caractérisent la thématique du présent numéro, la rédaction de Slavica Bruxellensia a donc procédé autrement que dans son entretien habituel, en menant une petite enquête sur l'érotisme et la sexualité. La table ronde virtuelle que nous avons " créée " a apporté un éventail de réponses que nous soumettons aux lecteurs sous la forme d'un entretien polyphonique. Il est intéressant d'observer combien les voix des intervenants se croisent, parfois s'opposent ou se répondent, bien qu'elles n'aient pas dialogué au sens strict du terme.

2 Avant d'entrer dans le vif du sujet, faisons un tour de table par ordre alphabétique des différentes voix qui interviennent:

Blažina Dalibor est professeur de littérature polonaise et de littérature comparée à l'Université de Zagreb ; 
Ulrike Jekutsch est professeur de littérature russe et de littérature comparée à l'Université de Greifswald ;

Maria Kistereva est doctorante en sciences du langage à l'Université Libre de Bruxelles et à l'Université Classique de Lisbonne ;

Wiera Meniok est professeur de littérature russe, polonaise et comparée ainsi que traductrice et directrice du Centre Scientifique Bruno Schulz à Drohobycz;

Joanna Partyka est professeur à l'Académie de Sciences Polonaises et à l'Université de Varsovie;

Jan Rubeš est spécialiste de littérature tchèque et professeur honoraire de l'Université libre de Bruxelles.

\section{Entretien}

SLAVICA BRUXELLENSIA : Quelle place occupent l'érotisme et la sensualité dans la littérature de votre pays?

Jan Rubeš : Il faut, avant toute autre chose, définir le terme. J'ai toujours essayé de distinguer quatre catégories de ce que cette question suggère: la sensualité, l'érotisme, le sexe et la pornographie. La première et la quatrième catégorie ne m'intéressent pas vraiment. La sensualité est partout où il y a le sentiment, le désir, la tentation et c'est un des moteurs de toute la littérature. Dès que deux personnes, le plus souvent du sexe opposé, se rencontrent (dans une œuvre littéraire), on peut supposer qu'il y aura une tension, soit négative, soit positive. Dans le second cas de figure, c'est celui qui correspond à notre investigation, la tension peut être plus forte encore si les protagonistes se rencontrent sans pouvoir réaliser leur désir de s'unir, et la sensualité devient ainsi un des sujets courant jusqu'à la banalité. Lisez (ou relisez) seulement "Fragments d'un discours" amoureux de Roland Barthes ${ }^{1}$. La correspondance amoureuse, l'éloignement forcé, la pression sociale, l'amour impossible, etc. on a de quoi remplir une immense bibliothèque. Le plus souvent, on entre dans l'intimité d'une personne qui se confesse. Une éditrice m'a dit récemment qu'elle lit en moyenne une vingtaine de manuscrits par semaine, dont dix-huit parlent de cela. "Moi, je vais vous raconter mon amour secret...» Bref, ce sont aujourd'hui, le plus souvent, des histoires destinées à un psy et, souvent, hélas, publiées.

Quant à la pornographie, elle est très rarement bonne et presque toujours commerciale. Qui plus est, elle ne fait pas rêver, ce qui est une des clés du succès de la littérature. Dans un roman, elle est moins visuelle que dans un film ou sur les photographies, bien plus explicites, tout en étant aussi tristes et à la longue même monotones.

Reste donc l'érotisme et le sexe, qui peuvent avoir une dimension métaphysique, face à la sensualité qui n'est que "méta » et à la pornographie qui n'est que "physique ».

Je dois avouer que je suis un mauvais lecteur de la littérature tchèque contemporaine, puisque je trouve son discours soit documentaire, soit naïf, voire enfantin. J'essaie de faire filtrer les livres par la passoire du temps et je ne lis que les livres que l'on m'offre (car ils plaisent à mes amis) ou que l'on me recommande. Pourtant, ils me tombent souvent des mains. J'aimerais donc mentionner deux écrivains, qui sont, pour un tas de raisons, difficiles à comparer, mais symptomatiques quant au traitement du sujet qui nous intéresse. Le temps et la condition de l'écrivain y sont pour beaucoup. 
Le premier est Milan Kundera (1929). Dans un entretien avec Bernand Pivot, au cours d'une de ses mémorables émissions « Apostrophes » (janvier 1984) ${ }^{2}$, il raconte qu'une lectrice lui a reproché son goût pour le sexe. Elle lui disait en substance : je voulais lire un écrivain qui a fui le régime totalitaire, $\mathrm{j}$ 'attendais donc quelque chose de profondément humaniste et je suis écœurée, car je trouve dans vos livres que du sexe, et encore du sexe. Sur ce, Kundera répond: finalement, le sexe ne m'intéresse que comme une façon de se comporter qui est fondamentalement individuelle. C'est dans tout ce qui précède l'acte même (qui en soi ne m'intéresse guère, dit-il) ou ce qui lui succède que je découvre le mystère d'un personnage qui est le héros de mon roman. Le sexe devient donc une fonction. Le second écrivain est Petra Hůlová (1979) ${ }^{3}$, de 50 ans sa cadette, ni exilée, ni confrontée au dilemme politique, libérée des contraintes idéologiques et existentielles. Chez elle on voit l'évolution de l'érotisme, propre aux romans de Kundera, au traitement du sexe. Sans parler d'obsession par le sexe, elle le rend cru, biologique, omniprésent, mais dans une langue à part. Je pense à Umělohmotný tř́pokoj (La Trichambre en plastique), écrit dans une langue riche, imagée, qui « littérarise » le sujet, celui de la confession d'une femme d'aujourd'hui dans un esprit d'autodérision. Un livre qu'il faut (se) traduire ou décoder, ce qui crée une distance au fond excitante entre les thèmes traités et la forme.

Il me semble d'ailleurs symptomatique que le sexe dans la littérature tchèque contemporaine est plus souvent traité par les femmes que par les hommes écrivains, passant souvent dans la catégorie pornographique. La vedette, sans être la pire, en est incontestablement Irena Obermannová, dont Le livre secret, décrivant ses aventures amoureuses avec Václav Havel peu avant son décès, a donné lieu à un débat plutôt populiste dans les milieux pseudo-intellectuels tchèques.

Ulrike Jekutsch : En Russie, l'érotisme et la sensualité sont devenus des sujets de la littérature post-pérestroïka. Et la littérature de ce type virait plutôt vers la pornographie. Depuis lors, l'érotisme est un sujet parmi les autres dans la littérature russe.

Maria Kistereva : Dans la littérature russe, l'érotisme et le sexe étaient des sujets tabous jusqu'à la chute de l'URSS : les œuvres ne pouvaient contenir que des allusions implicites à ce sujet. Après la pérestroïka, l'érotisme et la sensualité sont devenus conjoncturels, constituant dans les textes des auteurs contemporains une sorte d'anti-réaction à la tradition précédente, bien que souvent sans véritable adéquation avec la réalité.

Joanna Partyka : Parler de ce type de littérature m'est aisé en tant que spécialiste de la littérature ancienne. La littérature polonaise de la Renaissance et de l'époque baroque s'inscrivait parfaitement dans les courants et les modes européens, surtout dans le domaine de la poésie. L'érotisme et la sensualité étaient présents non seulement dans les œuvres rattachées au genre dit "erotyk», c'est-à-dire de « l'érotique », mais aussi dans les autres genres poétiques des " voluptés mondaines » ainsi que dans la poésie religieuse : c'était liée à « l'esprit de l'époque ». Les discours et plus particulièrement les discours de noce étaient imprégnés d'érotisme, bien que ce ne soit pas toujours perceptible aujourd'hui. Dans les traités d'éthique, dans la prose didactique et parénétique, dans les sermons, on luttait de façon acharnée contre "l'obscénité et la luxure» dans les habits et dans le comportement, dans les paroles et dans la littérature. Certaines auteurs, tels que Łukasz Górnicki, laissaient consciemment tomber les fragments indécents, voire trop érotiques, lorsqu'il 
traduisait des œuvres étrangères en polonais. D'un autre côté, ladite gaillardise de l'ancienne Pologne reposait toutefois dans une grande mesure sur le concept de cruc'est-à-dire de l'érotique.

Blažina Dalibor : Pour ce qui est de l'érotisme et de la sensualité dans la littérature croate contemporaine, on a l'habitude d'en parler de manières divergentes, voire franchement antagonistes. D'une part, certains critiques se plaignent de la "pudeur » des écrivains qui continuent à avoir des difficultés à dépasser les limites du tabou qui, étant issus de la culture croate traditionnelle, est ancré depuis longtemps. D'autres, pourtant, procèdent à l'inverse : considérant que la littérature croate a déjà dépassé ces limites depuis longtemps, en se référant au «butin » de cette littérature qui date des années 1960 (Alojz Majetić, Zvonimir Majdak, Ivan Slamnig), 1970 (Branimir Glumac) et surtout 1980, quand ces thématiques sexuelles étaient parfois mélangées avec un blasphème manifeste (Milko Valent, Borivoj Radaković) ou avec une ouverture sur d'autres champs de l'érotisme dans un sens féministe (Slavenka Drakulić) ou postmoderniste (Dubravka Ugrešić). Toutefois, il semblerait que la progressive "libération » du conservatisme "asexué » des mœurs, laquelle faisait à l'époque écho à une activité dans une certaine mesure d'opposition, ait été mise en question par l'apparition d'un changement de paradigme au début des années 1990 (l'agression et la guerre sur le territoire de la Croatie et des Balkans). Ce n'est sans doute qu'un peu plus tard, par esprit de résistance au paradigme officiellement promu de la littérature patriotique et martyrologique, mais aussi avec la volonté d'adopter les tendances postmodernistes, qu'est apparu tout un courant d'ouvertures de différentes sortes sur des sujets jusque-là étouffés : le sexe explicite et orgiaque (Stela Jelinčić, Damir Radić), l'érotique homosexuelle et les rapports sadiquesmasochistes (Dražen Ilinčić), l'amour lesbien (Marina Šur-Puhlovski). Ce sont des phénomènes qui peuvent être comparés à l'obsession dans les littératures issues d'une généalogie idéologique similaire, y compris à ses manquements, avec les littératures des pays de «l'Europe bis ", mais de façon un peu retardée eu égard à la censure du début des années 1990. Cependant, cela ne témoigne nullement de la qualité de cette littérature, qui se limite souvent à une vulgarisation (verbale) ou à un "art-pour-l'art-isme» sexuel. L'actualité de la littérature érotique est également confirmée par les nombreuses anthologies. Mais le vrai visage de la sensualité de cette littérature se trouve ailleurs : là où elle est présente de manière discrète ou symbolique.

Wiera Meniok : L'érotisme et la sensualité sont des composantes assez fortes de la littérature ukrainienne postmoderne. Dans cette littérature, les thématiques liées à l'érotisme se réalisent au moins dans trois champs: le conceptualisme (le roman Po'ovi doslidžja z ukrajinc'kogo seksu [Recherches de terrain sur le sexe ukrainien] d'Oksana Zabužko, le recueil de pièces 6 ho de Jurij Tarnavskij), le sexualisme (la prose de Jurij PokaL'̌̌uk et de Jurij Vinničuk), l'écriture de genre de type féminin (Evgenia Kononenko, Halina Pagutiak, Sofija Majdans'ka, Ludmila Taran ou Oksana Zabužko) et de type masculin (entre autres le groupe poétique « $\mathrm{Bu}-\mathrm{Ba}-\mathrm{Bu}$ » avec ses principaux représentants : Jurij Andruhovič, Vìktor Neborak et Oleksandr Irvanec').

Paru en ukrainien en 1996 mais non traduit en français, le roman Pol'ovi doslidžja z ukrajinc'kogo seksu de Zabužko est le premier livre majeur de la littérature ukrainienne dans lequel l'érotisme et la sexualité ont une signification conceptuelle. Dans la société postcommuniste, le titre même évoquait des connotations 
unanimement négatives, et telle était l'intention de l'auteure, étant donné qu'elle avait défini la conception du livre comme "une psychanalyse nationale postcommuniste", décrivant les destructions, les traumas et les ravages que le régime totalitaire et l'idéologie communiste ont provoqués dans le psychisme humain, entraînant une forme de médiocrité dans toutes les sphères de la vie de l'homme, y compris dans sa vie intime. Écrit dans un style réaliste, féroce, le livre a été reconnu comme le premier manifeste du féminisme dans la littérature ukrainienne. L'érotisme en tant qu'un certain argument du féminisme de Zabužko est également présent dans son recueil de nouvelles Sestro, sestro (Sœur, sœur, 2003). Aussi retrouvons-nous cet érotisme dans un des livres les plus célèbres que l'auteure ait écrit ces dernières années: Muzej Pokynutyh Sekretiv (Le musée des secrets abandonnés, 2009). Fortement ancré dans le féminisme, il constitue une extraordinaire épopée ukrainienne qui mène dans les recoins de la mémoire collective et individuelle, féminine et masculine, dans un espace-temps étendu et au cœur des événements historiques (de l'est de l'Ukraine à la Galicie, de l'entre-deuxguerres à nos jours). Malgré les fortes positions conceptuelles et féministes, l'érotisme prend dans ce livre une forme distincte de sexualité, grossie par le contexte historico-national qui est aujourd'hui très discuté et perçu de manières diverses : la pratique du sexe oral dans le repaire de l'UPA (Armée insurrectionnelle ukrainienne sous la direction de Stepan Bandera).

Vinničuk et PokaL'čuk sont irremplaçables en termes de description et de dramatisation du sexualisme dans la littérature ukrainienne postmoderne. Ils se positionnent toujours comme des écrivains contemporains, s'opposant clairement aux représentants de la « Génération '60 » qui sont politiquement engagés (Volodimir Javorivskij, Ivan Drač, Dmitro Pavličko, Roman Ivaničuk).

Pokalčcuk (1941-2008) est pour ainsi dire l'écrivain érotique culte en Ukraine, ce qui d'une part attire les lecteurs, mais d'autre part continue de susciter une vive critique. Parmi les romans sur la sexualité et l'érotisme, il faut mentionner Te, ščo na spodi (Ce qui est en-dessous, 1998), Čas prekrasnyj (Beau temps, 2002), Pamoročlyvyj zapah džungliv (L'étourdissante odeur de la jungle, 2005), Zaboroneni igry (Jeux interdits, 2005). On pourrait dire que dans les romans de PokaL'čuk, le sexe est montré dans sa forme première, sans tache, directe ; c'est pourquoi il est souvent touchant. La prose de Pokaǐčuk doit néanmoins plutôt être lue par un lecteur que ladite « vérité de la vie " n'irrite pas, ni n'effraie, car l'auteur parle de la sphère intime des relations interhumaines de manière très ouverte, provoquant constamment des tensions psychologiques.

Dans le domaine de la prose érotique, le concurrent le plus sérieux de PokaL'čuk est Vinničuk, l'auteur d'un roman à scandale sur les prostituées et les voleurs de Lviv, Divy noči (Nuits virginales, 1991), qui a encore paru à l'époque soviétique. Après, ont paru : Divy noči 2 (Nuits virginales 2), un court roman érotique intitulé Žytije garemnoje (Vie dans un harem) dans lequel l'auteur se moque du mythe national de la Roksolanka, ce qui provoqua un véritable scandale en Ukraine; un recueil de nouvelles Vesnjani igry $v$ osinnih sadah (Jeux printaniers dans les vergers d'automne). La courtoisie, la galanterie, l'ironie, le jeu intellectuel, la mystification sont les caractéristiques de la prose érotique de Vinničuk, dans laquelle l'histoire est habillée d'une métaphore de grande qualité artistique. Ses textes sont très intelligents, ses héros le sont aussi, ce qui permet de ne pas réduire cette prose à la dimension d'un érotisme brutal, qui est aussi présent dans la littérature ukrainienne contemporaine. 
Nous pouvons trouver de l'érotisme sous une forme dénaturée très concentrée dans les romans d'Olese Uljanenko (1962-2010), lequel cultive un érotisme brutal au travers d'un entourage laid, de ressentiments douloureux et de l'absence de sentiments. L'auteur voit en cela une esthétique et une sexualité spécifique. La vie sensuelle de ses personnages se passe dans le milieu des bandits, des prostituées, des meurtres, des rixes, des pillages: de part en part, elle est imprégnée d'un métaphorisme clairement dépressif. En 2009, le roman de Uljanenko Žinka jogo mriji (La femme de ses rêves) a été reconnu comme pornographique par la Commission Nationale pour la moralité : en conséquence, le livre a été retiré de la vente. Le roman a été réédité en 2010 et sa présentation s'est déroulée sous la forme d'une performance provocante dans une galerie commerciale de Kiev. Tam, de Pivden' (Là où est le Sud), le dernier livre publié du vivant de Uljanenko, reste lui aussi dans le genre de cet érotisme brutal. Celui-ci contient une catharsis idéologique cachée: une nostalgie pour l'accomplissement d'un érotisme et d'une sexualité rêvés dans la société postsoviétique qui a été condamnée à la médiocrité et à la brutalité dans les relations interhumaines.

SLAVICA BRUXELLensia : Estimez-vous qu'ils s'expriment/se développent de manière spécifique/différente selon qu'ils apparaissent dans la prose, la poésie ou le théâtre?

Ulrike Jekutsch : Je ne pense pas qu'il y ait des genres spécifiques pour la littérature érotique. Cela existe, bien entendu, mais l'érotisme peut s'écrire tant sous forme de poésie, de théâtre que de prose. Si je ne me trompe pas, la poésie érotique ainsi que la poésie d'amour sont des genres thématiquement définis.

Joanna Partyka : L'amour et l'érotisme étaient surtout présents dans la poésie bucolique et dans la poésie érotique, autrement dit dans les genres "spécialisés " dans ce domaine. L'élégie moderne du nouveau latin a aussi développé des formes d'élégie amoureuse liées à l'ars amandi et à l'amor puellae. Celles-ci rencontrèrent un grand succès auprès des poètes polonais. Mentionnons entre autres Kasper Twardowski, l'auteur des Lekcje Kupidynowe (Leçons de Cupidon), qui ont probablement été mises à l'index des livres interdits avant d'être publiées en 1617.

Maria Kistereva : La prose propose une approche analytique, la poésie une approche introvertie, alors que le théâtre s'exprime au travers des arts plastiques. Propres aux capacités de ces arts, ce sont les procédés dont ils disposent pour refléter l'érotisme. C'est avant tout la poésie, mais aussi la prose qui sont les plus adaptés pour exprimer l'érotisme. Le théâtre est un art qui est trop lié aux objets: la thématique peut devenir grossière.

Jan Rubeš : Je pense que le roman autobiographique ou faussement autobiographique véhicule plus souvent ce sujet dans la littérature tchèque contemporaine. Les quelques poètes que j'ai lus récemment semblent réservés et reflètent plus des préoccupations métaphysiques ou s'attachent à la description crue, voire cruelle du quotidien. Pour le théâtre, je me sens encore moins compétent car je ne vais que rarement en République tchèque et je ne fais pas partie des spectateurs assidus. Cependant, parmi les pièces que j'ai pu voir récemment, aucune n'avait une connotation sensuelle. À une exception près: l'adaptation du Charme discret de la bourgeoisie par le Théâtre Reduta de Brno, qui débordait de références au sexe dans sa dimension scatologique, dont on se souvient du film de Buñuel. Tout cela pour 
choquer le public et pour le faire rire. Car, comme on le sait, l'excès est dans l'art un moyen qui produit le grotesque.

Blažina Dalibor : En Croatie, il me semble que cette littérature a en fait un caractère secondaire par rapport à la littérature occidentale, surtout par rapport aux modèles de la littérature populaire, voire franchement commerciale. Mais dans le cadre de la littérature croate, elle crée sûrement certains modèles qui reflètent des phénomènes à caractère plus général : par exemple, l'obsession de la thématique du corps, la désintégration du modèle traditionnel de la vie sociale, la crise des valeurs et la fuite dans l'égoïsme, etc.

Wiera Meniok: Dans la littérature ukrainienne postmoderne, je pense que la littérature érotique a créé ses propres genres et ses propres modèles en prose, surtout grâce à PokaĽčuk, Vinničuk et Zabužko, mais aussi grâce aux positions extrêmes qui, dans ce contexte, appartiennent à Uljanenko. Dans le genre du roman érotique, il y a la création de Svetlana Povalaeva. Killer (2012), le premier recueil de nouvelles du jeune poète ukrainien Andrij Ljubka, apparaît comme curieux et non conventionnel dans le cadre de la prose érotique ukrainienne. Dans cette prose, l'auteur tisse des fantaisies érotiques, naïves et romantiques, mais aussi bestiales et ardentes.

Dans la poésie ukrainienne contemporaine, l'érotisme et la sensualité ont aussi leurs propres standards, bien que la thématique érotique soit plus sobre et pathétique dans la poésie, ce qui peut se remarquer dans les poèmes d'amour de PokaL'čuk et de Vinničuk, tout comme dans la poésie du poète et écrivain ukrainien culte Sergij Žadan. Nous les trouvons dans les poèmes érotiques sublimés dans la poésie des années 1990 de Andruhovič et de Neborak, chez l'éminent poète et l'un des meilleurs éditeurs ukrainiens d'aujourd'hui Ivan Malkovič, chez le jeune poète Dmitro Lazutkin, etc. La poésie n'a pas créé pour autant de genres ou de modèles autonomes, encore moins de courants liés à l'érotisme et à la sensualité, comme cela se perçoit dans la prose. Dans la poésie, l'érotisme a plutôt l'air sporadique, il est souvent une incrustation spécifique dans la poésie réflexive et philosophique. C'est pourquoi il n'est pas possible de citer un poète ou une poétesse dont la poésie se positionnerait comme érotique ou sensuelle.

Concernant l'écriture théâtrale ukrainienne contemporaine, c'est encore différent, car elle ne s'est pas développée de manière aussi diversifiée et plurielle que la prose et la poésie. En plus, les pièces des auteurs ukrainiens contemporains ne sont presque jamais présentées sur les scènes des théâtres. Il ne serait possible de citer que quelques pièces contenant des trames érotiques et sensuelles, sans être pour autant des manifestations d'érotisme. De telles pièces ont été écrites entre autres sous la plume de Pavel Arie, Neda Neždanova, Bohdan Žoldak, Lese Poderevjanski. La situation est encore complètement différente dans les théâtres ukrainiens contemporains, où les sujets érotiques et sensuels occupent souvent des positions dominantes : il suffit de mentionner le fameux acteur et metteur en scène Roman Vikitjuk et son propre théâtre érotico-sexuel, bien connu en Ukraine et en Russie.

SLAVICA BRUXELLENSIA : L'érotisme et la sensualité constituent-ils des références valables pour les discours théoriques sur la littérature, par exemple les discours postmoderniste, déconstructioniste, féministe et autres?

Joanna Partyka : Ils sont sûrement importants dans le discours féministe, et encore plus dans le postmodernisme. 
Maria Kistereva : Incontestablement, d'autant plus que l'érotisme et le désir sexuel trouvent souvent leur expression la plus absolue dans ces domaines.

Ulrike Jekutsch : Les chercheurs débattent, et ils publient des livres au sujet de l'érotisme dans la culture russe. Certains d'entre eux l'étudient dans le contexte de la déconstruction, du postmodernisme ou du féminisme. Je ne suis nullement experte dans ce domaine, mais cela ne me semble pas pertinent.

Blažina Dalibor : Oui, ces thématiques sont présentes dans le discours théorique. Par ailleurs, l'apparition du féminisme dans les années 1980, qui s'est ensuite développé en critique déconstructionniste et postmoderniste (Lada Čale Feldman, Nataša Govedić), ont joué un rôle important. À cet égard, il faut noter le rôle des recherches menées sur l'identité cachée de figures particulières appartenant à l'histoire de la littérature croate.

Wiera Meniok : Dans la théorie littéraire ukrainienne, les catégories de l'érotisme et de la sensualité sont prises en considération par le féminisme et les gender studies, qui sont toutefois de moins en moins populaires et actuels dans les recherches sur la littérature. Dans le contexte de cette méthode d'analyse littéraire, des travaux ont été écrits par Vera Ageeva, Tamara Hundorova, Solomija Pavličko, Nila Zborovska, Roksana Harčuk.

Jan Rubeš : Il ne faut certainement pas exclure une telle approche de la littérature tchèque contemporaine mais cela va mener le chercheur vers la sociologie de la littérature, la théorie du discours, la théorie de la réception, etc., donc vers la contextualisation des œuvres. En revanche, je trouve cet intérêt porteur de sens notamment dans l'analyse de la littérature et de l'art d'avant-garde. C'est là que l'on a fait exploser un tabou, sans le dé-tabouiser complètement, mais cela a ouvert de nouvelles voies. La littérature, il est vrai, restait plus confidentielle que la peinture : les tableaux de Jindřich Štyrský, de Toyen, les collages de Karel Teige, choquaient, et la Erotická revue que Štyrský faisait paraître au début des années 1930 était quasiment un samizdat secret. On savait que certains écrivains étaient auteurs d'œuvres déroutantes, Vítězslav Nezval ${ }^{4}$, et avant lui Guillaume Apollinaire ou Louis Aragon, mais elles étaient, à l'époque où je travaillais sur les avant-gardes, difficilement accessibles, surtout en Tchécoslovaquie. L'érotisme, le subconscient, le rêve, les travaux de Sigmund Freud auxquels on faisait référence - et ce qui servait parfois d'alibi - apportaient un regard nouveau au phénomène littéraire. Aujourd'hui, ces travaux sont faits et appartiennent à l'histoire de la littérature. Je ne crois pas qu'une approche similaire puisse décrypter une quelconque valeur essentielle dans la production littéraire tchèque d'aujourd'hui.

SLAVICA BRUXELlensia: Ces catégories sont-elles des repères constitutifs de vos recherches?

Joanna Partyka : Je travaille sur la culture de l'ancienne Pologne et ses différentes manifestations. L'érotisme est un aspect particulièrement important de cette culture, et j'y reviens souvent dans mes recherches, dernièrement en relation avec les manuels de confession et les livres de pénitence.

Ulrike Jekutsch : L'érotisme n'est certainement pas mon sujet. J'ai un peu écrit sur la sensualité ainsi que sur la poésie d'amour, mais je n'ai pas entrepris de travail sur l'érotisme. 
Maria Kistereva : Sont-elles des notions constitutives de mes recherches? Non.

Blažina Dalibor : Pas spécialement.

Wiera Meniok : Dans mes recherches, je ne m'intéresse pas à l'érotisme et à la sensualité quand ils se manifestent et sont présents dans les textes littéraires de manière évidente. Je m'occupe des sens plus cachés du texte littéraire, son intentionnalité et ses structures internes. Dans ce contexte, j'ai par exemple essayé d'examiner la prose poétique de Bruno Schulz et les poésies de Andruhovič du point de vue du plaisir du texte de Barthes et de la catégorie de l'érotisme qui y est liée, comprise comme une caractéristique de l'écrit du texte construit sur le principe des glissements, des failles, des non-dits, des réticences, des «vacillations du mystère » (Schulz).

L'érotisme et la sensualité manifestes dans la littérature ne s'est jamais trouvé, me semble-t-il, en première position dans le discours littéraire, et ce, indépendamment des déterminants nationaux ou historico-culturels de ce discours. Ces catégories n'en sont pas moins productives dans la littérature et elles reflètent souvent les étapes transitoires les plus intéressantes dans le développement de telle ou telle littérature nationale, comme c'est le cas dans la littérature ukrainienne depuis les années 1990 jusqu'à nos jours. Étant sortie du rideau de fer du réalisme socialiste et s'étant libérée des réalités communistes-soviétiques ainsi que des catégories de pensée imposées par le totalitarisme, la littérature ukrainienne recherche naturellement ce qui était autrefois interdit, notamment l'érotisme, la sexualité, la pornographie. Au travers de ces catégories, elle exprime d'une part la liberté retrouvée, mais d'autre part l'incapacité aussi de se réaliser pleinement dans cette liberté, bien que le fardeau des tabous et des clichés d'hier laisse une trace sombre sur ce qui aurait pu être beau et accompli, s'il n'avait pas été dévasté, réduit à la brutalité et au manque de perspective d'accomplissement.

Jan Rubeš : Cela a constitué, obligatoirement, un des repères de mes recherches dans les années 1970, quand j'ai écrit ma thèse sur le surréalisme. Mais il était exclu d'en parler ouvertement, même sous le couvercle de la science, dans le contexte castrateur du socialisme normalisé à la tchécoslovaque. Il faut aussi dire qu'à un certain âge, on trouve tous ces thèmes excitants car la littérature peut avoir une fonction aphrodisiaque. Je lisais, à vingt ans, le marquis de Sade, mais à cause de mon français fragile, je n'y comprenais pas grand-chose, ce qui n'était pas si mal car cela ouvrait un espace à l'imagination. Après, la plupart des œuvres semblent d'un érotisme plutôt « light », comme je trouve fade le Coca-Cola light. Cependant, une telle approche ne me semble plus vraiment révélatrice des phénomènes littéraires contemporains. Je travaille, comme bien d'autres collègues d'ailleurs, plus sur les topoï, sur des espaces croisés, sur les rencontres et sur les correspondances entre diverses formes d'art. Car je pense que contrairement aux chercheurs qui travaillent dans les domaines des sciences exactes et qui sont obligés de se spécialiser de plus en plus étroitement, nous, qui avons choisi les sciences humaines, évoluons du particulier au général. Et c'est dans cette vision large, toujours plus large, où les formes d'expression les plus diverses se confondent, que je trouve le plaisir de la culture, qui est devenue, pour moi, depuis longtemps, plus une passion qu'une profession. 


\section{ANNEXES}

Milan Kundera s'entretient avec Bernard Pivot (émission « Apostrophes », Antenne 2 [France], janvier 1984) (Lien externe)

\section{NOTES}

1. Barthes R., Fragments d'un discours amoureux, Seuil, Coll. Tel Quel, Paris, 1977, 280 p.

2. La vidéo de l'émission se trouve au bas de la page: "Apostrophes », janvier 1984 (France 2 [télévision publique française]) sur le site officiel de l'Institut National de l'Audiovisuel (http:// www.ira.fr).

3. Slavica Bruxellensia a publié un entretien avec Petra Hůlová dans son deuxième numéro (2009) : Flock S. \& Vacula R., « Entretien avec Petra Hůlová », in : Slavica bruxellensia [En ligne], nº 2, 2009, mis en ligne le 9 mai 2012. URL : http://slavica.revues.org/171 ; DOI : 10.4000/slavica.171.

4. Voir l'article de Sophie Teulière dans ce numéro : Teulière $S$., «Interdit et transgression dans Valérie ou la semaine des merveilles de Vítězslav Nezval », in : Slavica bruxellensia [En ligne], $\mathrm{n}^{\circ}$ 9, 2013, mis en ligne le 15 avril 2013. URL : http://slavica.revues.org/1341; DOI : 10.4000/ slavica.1341

\section{INDEX}

Index géographique : Croatie, Pologne, République tchèque, Russie, Tchécoslovaquie, Ukraine Mots-clés : érotisme, littérature comparée, littérature croate, littérature érotique, littérature polonaise, littérature russe, littérature tchèque, littérature ukrainienne oeuvrecitee PokaLčuk Ju. : Čas prekrasnyj, PokaLčuk Ju. : Pamoročlyvyj zapah džungliv, PokaLčuk Ju. : Te, ščo na spodi, PokaL'̌̌uk Ju. : Zaboroneni igry, Povalaeva Sv. : Killer, Tarnavskij Ju. : 6h0, Twardowski K. : Lekcje Kupidynowe, Uljanenko O. : Tam, de Pivden', Uljanenko O. : Žinka jogo mriji, Vinničuk Ju. : Divy noči, Vinničuk Ju. : Divy noči 2, Vinničuk Ju. : Vesnjani igry v osinnih sadah, Vinničuk Ju. : Žytije garemnoje, Zabužko O. : Muzej Pokynutyh Sekretiv, Zabužko O. : Pol'ovi doslidžja z ukrajinc'kogo seksu, Zabužko O. : Sestro, sestro

\section{AUTEURS}

\section{KATIA VANDENBORRE}

Université Libre de Bruxelles (Belgique) et Université de Varsovie (Pologne) ; membre du comité de rédaction de « Slavica Bruxellensia » 
DOROTA WALCZAK

Responsable de la Chaire de Polonais, section de Langues et Littératures modernes, option Slaves, de l'Université Libre de Bruxelles (ULB, Belgique) Unité de recherche PHILIXTE « Études littéraires, philologiques et textuelles » (ULB) Rédactrice en chef de Slavica Bruxellensia

\section{BLAŽINA DALIBOR}

Professeur de littérature polonaise et de littérature comparée à l'Université de Zagreb

\section{ULRIKE JEKUTSCH}

Professeur de littérature russe et de littérature comparée à l'Université de Greifswald

\section{MARIA KISTEREVA}

Doctorante en sciences du langage à l'Université Libre de Bruxelles et à l'Université Classique de Lisbonne

WIERA MENIOK

Professeur de littérature russe, polonaise et comparée ainsi que traductrice et directrice du Centre Scientifique Bruno Schulz à Drohobycz

\section{JOANNA PARTYKA}

Professeur à l'Académie de Sciences Polonaises et à l'Université de Varsovie

\section{JAN RUBEŠ}

Professeur honoraire de l'Université libre de Bruxelles, spécialiste de littérature tchèque 